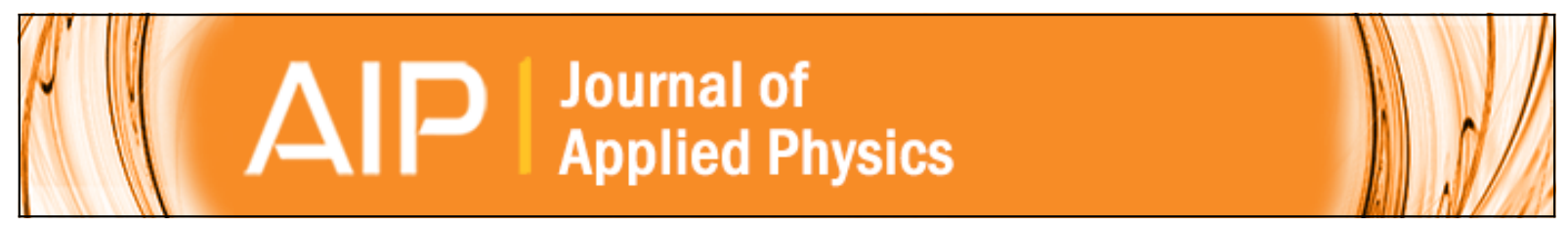

\title{
High temperature stress-induced "double loop-like” phase transitions in Bi-based perovskites
}

K. G. Webber, Y. Zhang, Wook Jo, J. E. Daniels, and J. Rödel

Citation: Journal of Applied Physics 108, 014101 (2010); doi: 10.1063/1.3428373

View online: http://dx.doi.org/10.1063/1.3428373

View Table of Contents: http://scitation.aip.org/content/aip/journal/jap/108/1?ver=pdfcov

Published by the AIP Publishing

\section{Articles you may be interested in}

High pressure-induced phase transitions in CdS up to 1 Mbar

J. Appl. Phys. 113, 083509 (2013); 10.1063/1.4792233

High pressure behavior of BiMn 205

AIP Conf. Proc. 1512, 100 (2013); 10.1063/1.4790930

Structural phase transition and physical properties of tetragonal $0.85 \mathrm{~Pb}$ ( Zn $1 / 3 \mathrm{Nb} 2$ / 3 ) O $3-0.15 \mathrm{PbTiO} 3$ single crystals

J. Appl. Phys. 106, 074110 (2009); 10.1063/1.3238249

Discontinuous temperature-dependent macroscopic strain due to ferroelastic domain switching and structural phase transitions in barium strontium titanate

Appl. Phys. Lett. 91, 192908 (2007); 10.1063/1.2809388

Effects of the substitution of $\mathrm{Al}$ for Fe on phase transition, crystal structures, and magnetic properties of $\mathrm{Nd} 3$ ( $\mathrm{Fe}$ , Ti ) 29 -type intermetallics

J. Appl. Phys. 100, 103910 (2006); 10.1063/1.2386945

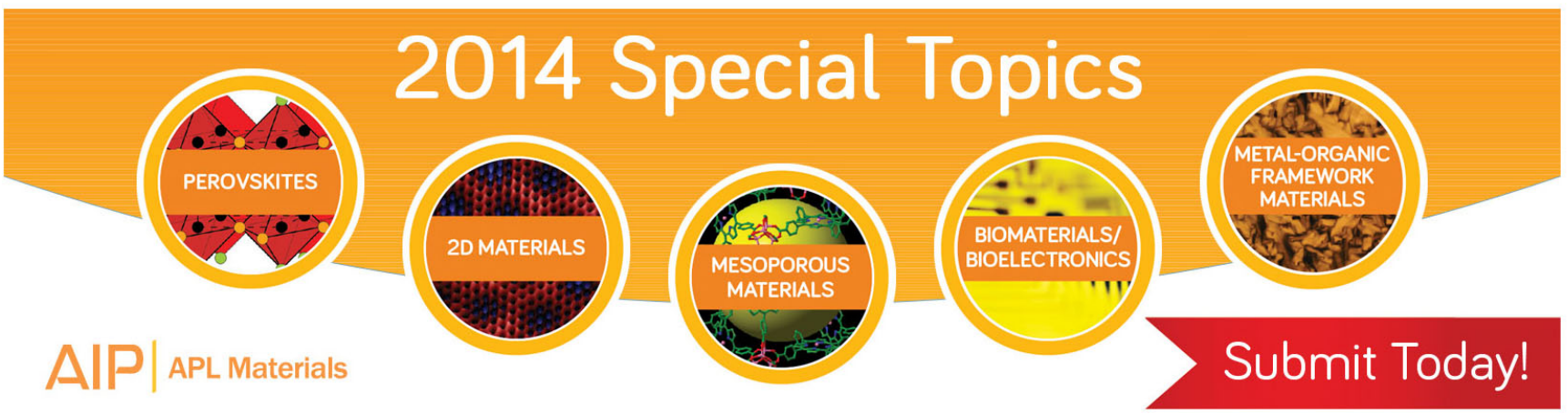




\title{
High temperature stress-induced "double loop-like" phase transitions in Bi-based perovskites
}

\author{
K. G. Webber, ${ }^{1, a)}$ Y. Zhang, ${ }^{1}$ Wook Jo, ${ }^{1}$ J. E. Daniels, ${ }^{2,3}$ and J. Rödel ${ }^{1}$ \\ ${ }_{1}^{1}$ Institute of Materials Science, Technische Universität Darmstadt, 64287 Darmstadt, Germany \\ ${ }^{2}$ European Synchrotron Radiation Facility, F-38043 Grenoble, France \\ ${ }^{3}$ School of Materials Science and Engineering, University of New South Wales, 2052 Sydney, Australia
}

(Received 11 March 2010; accepted 17 April 2010; published online 2 July 2010)

\begin{abstract}
Polycrystalline $0.94\left(\mathrm{Bi}_{1 / 2} \mathrm{Na}_{1 / 2}\right) \mathrm{TiO}_{3}-0.06 \mathrm{BaTiO}_{3}$ samples were tested under uniaxial mechanical compression at various temperatures in the vicinity of the polar tetragonal to nonpolar tetragonal phase boundary. They are shown to display double loop-like stress-strain behavior, marked by a closed ferroelastic hysteresis loop. Thus, it forms a mechanical analog to the polarization-electric field hysteresis behavior of barium titanate above the Curie temperature. As temperature is increased there is an apparent loss of macroscopically observable ferroelasticity, despite the persistence of tetragonality. Macroscopic experimental results are discussed in conjunction with temperature-dependent and stress-dependent high-energy x-ray diffraction data. This reveals a phase transition below the Curie temperature, marked by a discontinuous change in lattice parameters and octahedral tilting during compressive mechanical loading. () 2010 American Institute of Physics. [doi:10.1063/1.3428373]
\end{abstract}

\section{INTRODUCTION}

Ferroelectrics (FEs) are a technologically important class of materials utilized in numerous engineered structures and systems. Historically, due to the excellent material properties and an acceptable Curie temperature, lead zirconate titanate (PZT) has been the material of choice. However, increasing government regulations, which limit the use of toxic substances such as lead, have increased pressure to find an environmentally safe alternative. ${ }^{1} \mathrm{Bi}_{1 / 2} \mathrm{Na}_{1 / 2} \mathrm{TiO}_{3}(\mathrm{BNT})$, originally discovered by Smolenskii and co-workers ${ }^{2}$ nearly 50 years ago, is an appealing lead-free FE alternative because of its large remanent polarization. ${ }^{3}$ However, BNT suffers from a large coercive field $\left(E_{\mathrm{C}}=73 \mathrm{kV} \mathrm{cm}^{-1}\right)^{4}$ at room temperature as well as a rhombohedral to tetragonal phase transition below the Curie point, ${ }^{5}$ both of which limit its usefulness in the transducer industry.

Solid solutions of $(1-x)\left(\mathrm{Bi}_{1 / 2} \mathrm{Na}_{1 / 2}\right) \mathrm{TiO}_{3}-x \mathrm{BaTiO}_{3}$ $(\mathrm{BNT}-100 x \mathrm{BT})$ near the morphotropic phase boundary $(0.06 \leq x \leq 0.07)$ have shown a reduced coercive field $\left(30 \mathrm{kV} \mathrm{cm}^{-1}\right){ }^{3}$ in addition to a large remanent polarization at room temperature ${ }^{3,6}$ and the appearance of large unipolar strains at elevated temperatures. ${ }^{6}$ Initial investigations of $\mathrm{BNT}-100 x \mathrm{BT}$ claimed that there was a high temperature $\left(150 \leq T \leq 200{ }^{\circ} \mathrm{C}\right) \mathrm{FE}$ to anti-FE (AFE) phase transition for compositions that contained less than approximately $10 \mathrm{BT}{ }^{4}$ in disagreement with more recent neutron powder diffraction measurements that show a rhombohedral $(R 3 c)$ to tetragonal $(P 4 b m)$ phase transition in BNT below the Curie temperature. ${ }^{7}$ In the work by Takenaka et al., ${ }^{4}$ the appearance of a pinched polarization-electric field hysteresis loop observed in polycrystalline BNT-5BT at elevated temperatures was taken to be the result of a FE-AFE transformation. However, considering that the pinching in polarization hys-

\footnotetext{
a)Electronic mail: webber@ceramics.tu-darmstadt.de.
}

teresis can be caused by many different mechanisms such as relaxor to $\mathrm{FE}$ transition, ${ }^{8} \mathrm{AFE}$ to $\mathrm{FE}$ transition, ${ }^{9}$ or severe aging in FEs, ${ }^{10}$ the identity of the high temperature phase in the BNT-100xBT system remains unclear.

Despite their interesting and complex phase structures, to date there has been very few studies of the mechanical behavior of solid solutions of BNT-BT. ${ }^{11}$ Such studies are vital for their implementation into devices. The following study presents macroscopic ferroelastic hysteresis curves for polycrystalline BNT-6BT at various temperatures from 25 to $300{ }^{\circ} \mathrm{C}$. A unique stress-induced high temperature phase transition behavior not previously observed in polycrystalline FEs is presented and rationalized through an in situ high-energy $\mathrm{x}$-ray diffraction (XRD) analysis done under compressive stresses at elevated temperature.

\section{EXPERIMENTAL DETAILS}

Solid solutions of BNT-6BT were prepared using the solid oxide processing method. The oxide precursors were reagent-grade oxide and carbonate powders: $\mathrm{Bi}_{2} \mathrm{O}_{3}$ (Alfa Aesar, 99.975\%), $\mathrm{TiO}_{2}$ (Alfa Aesar, 99.9\%), $\mathrm{Na}_{2} \mathrm{CO}_{3}$ (Alfa Aesar, 99.5\%), and $\mathrm{BaCO}_{3}$ (Alfa Aesar, 99.8\%). Each was weighed according to the stoichiometric formula and ballmilled for $24 \mathrm{~h}$ in an ethanol suspension followed by drying at $110{ }^{\circ} \mathrm{C}$. Calcination of the powder was performed at $900{ }^{\circ} \mathrm{C}$ for $3 \mathrm{~h}$. After calcination, the batch was again ballmilled, dried, and ground to ensure a fine, uniform powder. Pressed cylindrical disks of $20 \mathrm{~mm}$ in diameter and about 8.5 $\mathrm{mm}$ in thickness were then cold isostatically pressed in a silicone oil bath under a pressure of $350 \mathrm{MPa}$ and sintered at $1150{ }^{\circ} \mathrm{C}$ for $3 \mathrm{~h}$ in an air atmosphere. Following sintering, the disks were ground to the final thickness, which was not identical for the XRD and mechanical experimentation.

High energy XRD experiments were carried out at beamline ID15A of the European Synchrotron Radiation Fa- 


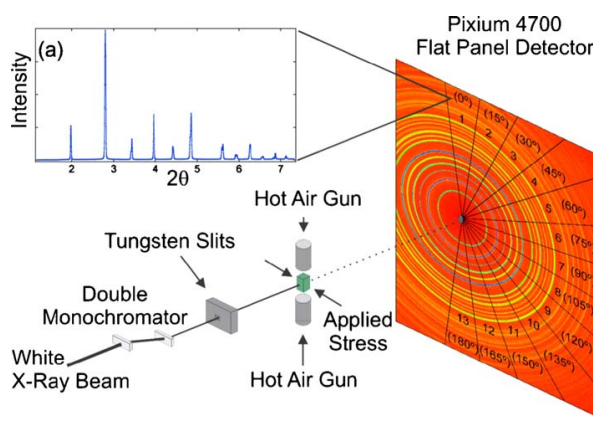

FIG. 1. (Color online) Schematic diagram of the XRD setup.

cility. A beam energy of $68.02 \mathrm{keV}$ was selected using a double Laue monochromator and beam size set to 0.3 $\times 0.3 \mathrm{~mm}^{2}$ using a set of tungsten slits. A sample of dimensions $1 \times 1 \times 1.6 \mathrm{~mm}^{3}$ was placed under compression using a BOSE electroforce test bench. The sample temperature was controlled to a thermocouple in contact with the sample surface using a double hot air gun blowing on opposite sides of the sample. XRD patterns were recorded using a Pixium 4700 flat panel detector ${ }^{12}$ at a distance of $882.2 \mathrm{~mm}$ from the sample. A schematic diagram of this setup is shown in Fig. 1. Using this geometry diffraction patterns with scattering vectors aligned both parallel and perpendicular to the loading direction can be isolated. ${ }^{13}$ Temperature dependent XRD measurements were done separately with the same equipment from room temperature to $208{ }^{\circ} \mathrm{C}$.

For the determination of the exact wavelength and instrumental parameters, $\mathrm{LaB}_{6}$ profile was recorded and refined using the software package FULLPROF. ${ }^{14}$ The Bragg peaks were modeled with the built-in Thompson-Cox-Hastings pseudo-Voigt function and the background was fit with a linear interpolation among selected points. The zero angular shift, scale factor, lattice parameters, and atomic parameters were refined. The refinements were carried out sequentially from low to high temperature data so that quick and reliable convergence was achieved. Since the noncubic distortion of the low temperature $P 4 \mathrm{bm}$ phase was already too small to be distinguished from cubic symmetry in a straightforward manner, changes in the intensity ratio of the pseudocubic (200) to (110) and (111) were traced to detect the phase transition. The choice of the space group for the detected new phase was made based on group-subgroup relations.

Cylindrical samples used for macroscopic stress-strain measurements, with a final diameter of $5.9 \mathrm{~mm}$ and height of $6 \mathrm{~mm}$, were core-drilled from the sintered, ground disk. Before testing, each specimen was annealed at $400{ }^{\circ} \mathrm{C}$ for $1 \mathrm{~h}$ and cooled with a rate lower than $1{ }^{\circ} \mathrm{C} \min ^{-1}$ to room temperature to remove potential residual stresses. This annealing step was also preformed between mechanical compression tests to relieve residual stresses and depole the previously tested samples. The macroscopic ferroelastic behavior of unpoled BNT-6BT samples was characterized on a $10 \mathrm{kN}$ screw-type load frame (Zwick, Z010). The longitudinal strain of a sample located inside of a heating chamber (accuracy of $+/-0.1^{\circ} \mathrm{C}$ ) was measured by a linear variable differential transducer (LVDT) capable of resolving displacements of approximately $10 \mathrm{~nm}$. The LVDT was situated out-

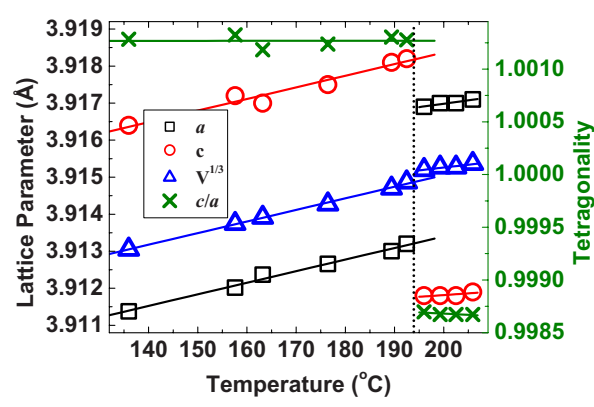

FIG. 2. (Color online) Changes in the lattice parameters, the averaged linear expansion, and $c / a$ of the tetragonal structure for polycrystalline BNT-6BT as a function of temperature. A discontinuous change in all the parameters presented is clearly discerned at about $195^{\circ} \mathrm{C}$, which is analyzed to be due to a $P 4 \mathrm{~mm}$ to $P 4 / \mathrm{mmm}$ phase transition. (The size of symbols used approximately reflects $95 \%$ confidence interval for each fitted peak.)

side of the heated region. A detailed description of the experimental setup can be found elsewhere. ${ }^{15}$ During testing short-circuited specimens were compressively loaded to a maximum uniaxial stress of $-385 \mathrm{MPa}$ with a linear loading/ unloading rate of $-3.7 \mathrm{MPa} \mathrm{s}^{-1}$.

\section{RESULTS AND DISCUSSION}

\section{A. Temperature-dependent structure}

A temperature dependent high-energy XRD study in conjunction with permittivity measurements was performed on BNT-6BT ceramics. In agreement with previous diffraction studies by Jones and Thomas ${ }^{7}$ on pure BNT and the permittivity measurements by Hiruma et al. ${ }^{16}$ on BNT-based systems, it was observed, based on temperature-dependent permittivity measurements, that the room temperature $R 3 \mathrm{~m}$ rhombohedral phase ${ }^{17}$ evolved into a $P 4 \mathrm{~mm}$ tetragonal phase at the depolarization temperature of $87{ }^{\circ} \mathrm{C}$. In contrast to the previous studies, ${ }^{7,16}$ however, a further investigation above the depolarization temperature up to $208^{\circ} \mathrm{C}$ revealed that there is another structural transition before the structure evolves into the cubic $P m \overline{3} m$. Interestingly, this transition takes place at the temperature where the frequency dispersion in the permittivity vanishes. Combined with the group theory, this high temperature phase was determined to be $P 4 / \mathrm{mmm}$, whose point group is $4 / \mathrm{mmm}$, which is no longer ferroelectrically active. In fact, this type of transition from FE to noncubic paraelectric was also reported in pure BNT through transmission electron microscopy. ${ }^{18,19}$ It was suggested that the nature of the transition be a second order whose microscopic state changes from FE-ferroelastic to paraelectric-ferroelastic, which will be one of the major points to be discussed in depth within this paper.

The structural information around the transition temperature obtained by Rietveld refinements is summarized in Fig. 2 . It is seen that a drastic change in all the parameters occurs at the transition temperature of $195^{\circ} \mathrm{C}$. Below this boundary both the $c$ and $a$ lattice parameters are increasing. However, at the phase transition temperature $c$ sharply decreases while $a$ sharply increases, resulting in a discontinuous increase in the unit cell volume represented by the changes in the volume averaged pseudocubic lattice parameter of $P 4 \mathrm{~mm}$ (3.1 $\left.\times 10^{-5} \AA /{ }^{\circ} \mathrm{C}\right)$ and $\mathrm{P} 4 / \mathrm{mmm}\left(1.5 \times 10^{-5} \AA /{ }^{\circ} \mathrm{C}\right)$. The most 

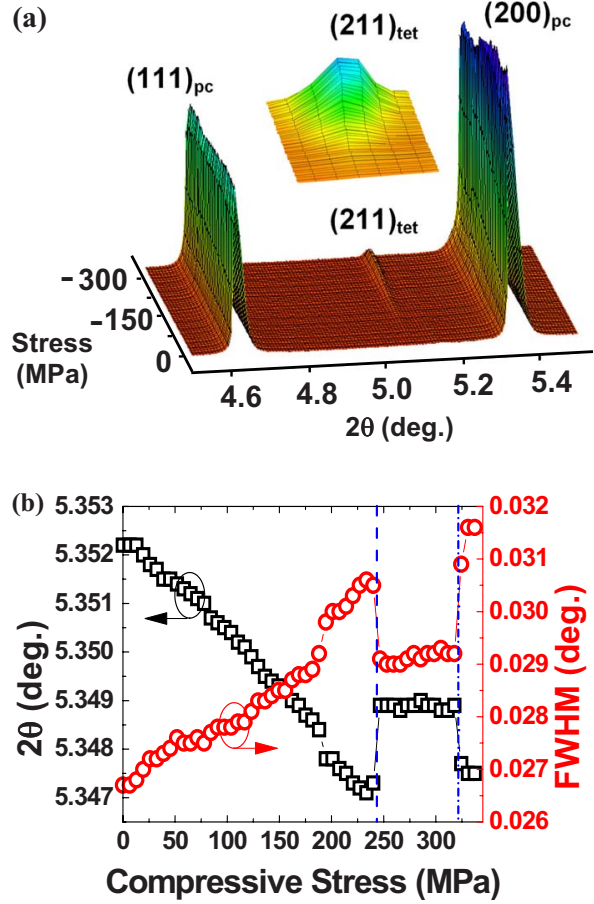

FIG. 3. (Color online) Surface plot (a) showing the evolution of selected XRD peaks for BNT-6BT perpendicular to the applied uniaxial stress. Due to the relatively weak intensity of the (211) tet superlattice reflection, a magnified view of the peak is presented (b) illustrating the changes in the position and FWHM of (200) $)_{p c}$ reflection as a function of stress.

distinctive difference between $P 4 \mathrm{~mm}$ and $P 4 / \mathrm{mmm}$ in the currently investigated system exists in their $c / a$ ratios, which are larger and smaller than 1 , respectively. Note that the $\mathrm{P} 4 / \mathrm{mmm}$ phase is supposed to be paraelectric-ferroelastic in terms of the point group but develops a negative spontaneous strain during the transition from $P m \overline{3} m$ to $P 4 / m m m$. This is not common for most ferroelastics whose $c / a$ ratio is larger than 1 .

\section{B. Stress-dependent structure}

To elucidate the effect of uniaxial stress on phase stability and structural changes, in situ high-energy XRD was performed at $200{ }^{\circ} \mathrm{C}$, slightly higher than the phase transition temperature $\left(195^{\circ} \mathrm{C}\right)$ of the stress-free specimen. It was assumed during analysis that the macroscopic and microscopic stress states were equivalent. In polycrystalline materials, however, grain-to-grain interactions and inhomogeneities can create fluctuations in the stress field between various grains. Figure 3(a) shows a surface plot of a selected $2 \theta$ region. At approximately $-150 \mathrm{MPa}$ the $(211)_{\text {tet }}$ superlattice reflection, which is characteristic of the presence of oxygen octahedral tilting, appears and its intensity increases continuously with stress. The fact that the stress easily converts $P 4 / \mathrm{mmm}$ into $P 4 / \mathrm{mbm}$ and at higher stress levels into $P 4 \mathrm{bm}$ indicates that there should be a phase boundary separating the systems with and without oxygen octahedral tilting at $x<0.06$, since the compositions with a lower BT content are known to exhibit oxygen octahedral tilting. ${ }^{17}$ More importantly, the continuous change in the intensity clearly implies continuous tilting of the oxygen octahedra, which is expected to be rep- resented by a change in the stress-strain curve around the stress level of $-150 \mathrm{MPa}$. A further increasing stress level up to about $-250 \mathrm{MPa}$ is seen to induce a nonpolar-polar phase transition, which is apparent from a discontinuous change in the intensity ratio between $(111)_{p c}$ and $(200)_{p c}$ as well as a development of an anisotropic $(200)_{p c}$.

To better identify the stress-induced structural changes the peak fitting module in the commercial software ORIGIN was employed for single peak profile fitting of the $(200)_{p c}$ reflection from the diffraction patterns collected along the direction perpendicular to the applied stress direction. Due to the very small tetragonal distortion of the given system, all the $(200)_{p c}$ peaks were assumed to be a single peak, and the $2 \theta$ position and full-width-at-half-maximum (FWHM) value were determined using a pseudo-Voigt function. The results of this analysis are shown in Fig. 3(b). These two parameters were chosen to reveal the effect of stress on the lattice distortion (2 $\theta$ values) and tetragonality or lattice stress (FWHM) of the pseudocubic system. Three distinctive critical stress levels, where both parameters changed abruptly, were approximately: $-190 \mathrm{MPa}$ (CS1), -240 MPa (CS2), and $-320 \mathrm{MPa}(\mathrm{CS} 3)$. The rate of change in both parameters up to CS2 remained relatively constant, despite the discontinuity observed at CS1. The decrease in the peak position, which indicates an increase in the average volume, was accompanied by an increase in FWHM. This implies that a macroscopically detectable strain induced by the stress is primarily volumetric up to CS2. At CS2, a significant contraction in the unit cell volume and decrease in FWHM are noted, which strongly indicates a phase transition at this stress level. From the intensity ratio between $(111)_{p c}$ and $(200)_{p c}$ as well as the presence of the $(211)_{t e t}$ superlattice reflection, the space group of the transformed phase can be assigned as $P 4 \mathrm{bm}$. It is interesting to note that a volumetric change was not observed in the $\mathrm{P} 4 \mathrm{bm}$ phase due to an external uniaxial stress. This is evident from the constant $2 \theta$ and FWHM values measured between CS2 and CS3. At stresses above CS3 the system developed a strong texture and a sharp increase in tetragonality, indicated by the presence of a noticeable anisotropy in the $(200)_{p c}$ peak. At the maximum stress, approximately $-340 \mathrm{MPa}$, the sample fractured. It was therefore not possible to measure the diffraction patterns during unloading.

\section{Macroscopic experimental measurements}

The macroscopic ferroelastic constitutive behavior for unpoled BNT-6BT at various temperatures up to $300{ }^{\circ} \mathrm{C}$ was characterized under uniaxial compressive stress. Representative hysteresis curves at $25,190,200$, and $215^{\circ} \mathrm{C}$ are presented in Fig. 4. At room temperature polycrystalline BNT-6BT displays typical ferroelastic behavior. During initial loading the sample response is linear to the applied compressive stress. However, at a critical stress level, defined here as the onset stress, $\sigma_{\mathrm{o}}$, domains begin to ferroelastically reorient, marked by a nonlinear change in the tangent modulus. The onset stress is found by a tangent-intersection method shown explicitly in Fig. 4(a). The onset stress at room temperature for BNT-6BT was measured as approxi- 


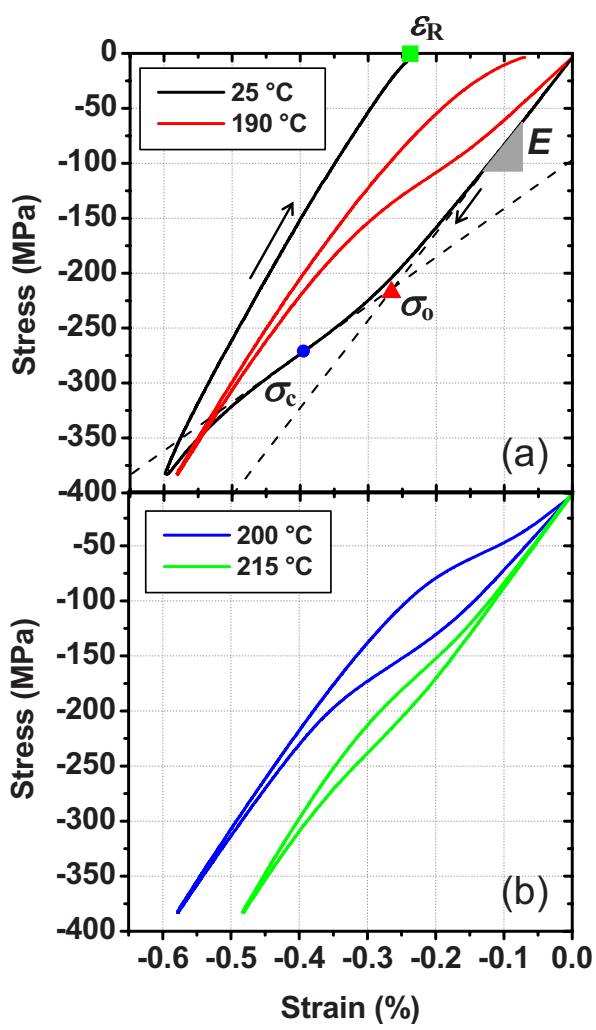

FIG. 4. (Color online) Stress-strain hysteresis curves for BNT-6BT polycrystalline specimens at various temperatures. The coercive stress (blue dot), onset stress (red triangle), remanent strain (green square), and initial compliance $\left(s_{33}=1 / E\right)$ are labeled on the ferroelastic hysteresis curve at $25^{\circ} \mathrm{C}$; the direction of loading is indicated by the inset arrows.

mately $-212 \mathrm{MPa}$. As the compressive stress is increased the switching rate maximizes at the inflection point, defined as the coercive stress, $\sigma_{\mathrm{c}}$. Upon unloading from the maximum stress the material behavior appears initially linear. This linear regime is followed by nonlinear material behavior principally caused by domains reorienting back to their original state due to local internal electrical and mechanical fields. This deviation from linearity is referred to as backswitching. When the compressive stress is removed a remanent strain, $\varepsilon_{R}$, remains due to domains that have remained in the ferroelastically reoriented directions.

High temperature compressive loading experiments of polycrystalline BNT-6BT above the phase transition temperature previously shown in Fig. 2, which separates the polar tetragonal $P 4 \mathrm{~mm}$ and the nonpolar tetragonal $P 4 / \mathrm{mmm}$ phases, show closed loop stress-strain behavior [Fig. 4(b)]. It is assumed that a high temperature stress-induced phase transition between these two space groups, as shown to occur at $200{ }^{\circ} \mathrm{C}$ due to compressive mechanical loading in Fig. 3, was responsible for this closed hysteresis behavior, similar to the paraelectric-FE electric field-induced phase transition in BT above the Curie temperature. ${ }^{20,21}$ Please note for comparison to high temperature double loop behavior in $\mathrm{BT}$ that the independent variable (electric field) is located on the abscissa, while the polarization is on the ordinate. As temperature was increased from $25{ }^{\circ} \mathrm{C}$ the macroscopic remanent strain decreased to zero above approximately $200{ }^{\circ} \mathrm{C}$. In the region between 200 to $220{ }^{\circ} \mathrm{C}$ the specimens exhibited a

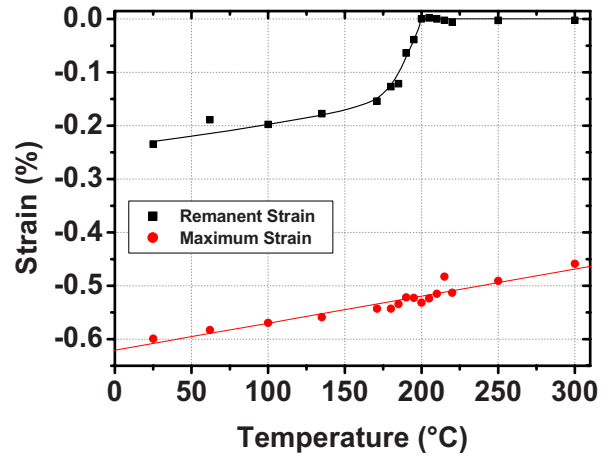

FIG. 5. (Color online) Remanent and maximum strain as a function of temperature for polycrystalline BNT-6BT samples loaded under uniaxial compression to a maximum stress of $-385 \mathrm{MPa}$.

closed (double loop) hysteresis. The term double hysteresis loop was originally used to describe the electric field induced cubic-tetragonal phase transformation in barium titanate above the Curie temperature. ${ }^{21}$ However, in the case of a stress-induced phase transition it is not possible to apply tensile stresses large enough to develop both lobes of the double loop. At stress levels above the phase transition stress the material behavior was linear. Due to the applied stress the domains transforming from $P 4 / \mathrm{mmm}$ to $P 4 \mathrm{bm}$ had a preference for crystallographic orientation $90^{\circ}$ to the applied stress. This resulted in an automatic loss of domains available to be ferroelastically switched in the $P 4 \mathrm{bm}$ phase upon completion of the stress-induced phase transition. Experiments at temperatures above $220{ }^{\circ} \mathrm{C}$ did not show closed loop behavior because the maximum compressive stress $(-385 \mathrm{MPa})$ was no longer large enough to initiate a phase transformation. Experiments of BNT-6BT at $250{ }^{\circ} \mathrm{C}$, well within the stressfree $P 4 / \mathrm{mmm}$ region between the high temperature cubic phase $(P m \overline{3} m)$ and the lower temperature stress-free $P 4 m m$ phase, show a linear stress-strain behavior despite the ferroelasticity expected from the point group symmetry. For this reason $P 4 / \mathrm{mmm}$ will be referred to as a potential ferroelastic.

The thermal effect on the macroscopic strain was quantified at numerous temperatures, as shown in Fig. 5. At temperatures below approximately $185{ }^{\circ} \mathrm{C}$ there was an apparent linear decrease in remanent strain with increasing temperature. However, at temperatures above $185^{\circ} \mathrm{C}$ the decrease in remanent strain became nonlinear until approximately $200{ }^{\circ} \mathrm{C}$, where the remanent strain vanished. This temperature corresponds well to the $P 4 / \mathrm{mmm}-P 4 \mathrm{~mm}$ stressfree phase transition temperature determined by XRD (Fig. 2 ). It appears from the macroscopic ferroelastic measurements that the reduction in remanent strain was continuous. In contrast, previous high temperature ferroelastic measurements of PZT displayed a linear decrease in remanent strain to the depolarization temperature $\left(T_{\mathrm{D}}=250{ }^{\circ} \mathrm{C}\right)$ where it remained zero up to the Curie temperature $\left(T_{\mathrm{C}}=300^{\circ} \mathrm{C}\right) .{ }^{15}$ Experimental measurements between the Curie temperature and the $P 4 \mathrm{~mm}-\mathrm{P} 4 / \mathrm{mmm}$ phase transition temperature (e.g., 250 and $300{ }^{\circ} \mathrm{C}$ ) did not display a remanent strain. There are two possible explanations for this behavior. First, the critical stress required to ferroelastically reorient domains is outside 
of the applied stress regions used during experimentation, either less than $-3.7 \mathrm{MPa}$ or greater than $-385 \mathrm{MPa}$. Previous studies on lanthanum-doped bismuth ferrite-lead titanate $\left(\mathrm{BiFeO}_{3}-\mathrm{PbTiO}_{3}\right)^{22}$ have shown an increase in switchability with decreasing $c / a$ ratio, indicating that the critical stress in the $P 4 / \mathrm{mmm}$ phase may be lower than the applied preload $(-3.7 \mathrm{MPa})$. Second, that $P 4 / \mathrm{mmm}$ is in fact paraelectric-paraelastic. However, permittivity measurements clearly show an additional dielectric anomaly at around $278{ }^{\circ} \mathrm{C}$. Such an anomaly is known to be related to the transition to the paraelectric-paraelastic cubic $P m \overline{3} m$, indicating that $P 4 / \mathrm{mmm}$ should be ferroelastic.

The maximum strain, obtained at the maximum stress of $-385 \mathrm{MPa}$, does not show the same nonlinear decreasing trend with temperature increasing through the $P 4 m m-P 4 / m m m$ phase transition. Maximum strain values, instead, show an apparent linear decrease up to the maximum temperature of $300{ }^{\circ} \mathrm{C}$. As temperature is increased the energy threshold for ferroelastic switching decreases potentially increasing the number of domains that can be mechanically reoriented. The lower energy barrier for switching, however, is offset by a simultaneous decrease in the spontaneous strain of the unit cell, which has been shown to decrease the macroscopic remanent and maximum strains during uniaxial compressive loading. In BNT-6BT, as previously observed in PZT, ${ }^{15}$ there was a linear decrease in maximum strain throughout the temperature range examined. Interestingly, this same trend in maximum strain is observed in BNT-6BT despite the high temperature phase transition at the $P 4 \mathrm{~mm}-\mathrm{P} 4 / \mathrm{mmm}$ boundary around $195{ }^{\circ} \mathrm{C}$. It appears that the decreasing tetragonality was offset by the additional strain increase during the stress-induced $P 4 m m m-P 4 b m$ phase transition, keeping the maximum strain values stable. It is also important to note that the reference strain used to determine the remanent and maximum strain values was at the temperature under consideration with a preload of -3.7 $\mathrm{MPa}$. It is likely that if the decrease in strain during the heating cycle were considered the maximum strain would show an anomaly at the phase transition temperature.

The stability of the mechanical properties of BNT-6BT was measured as a function of temperature in terms of the effective compliance and characteristic stresses, as shown in Fig. 6. In the lower temperature $P 4 \mathrm{~mm}$ phase the compliance was found to increase nonlinearly with increasing temperature, where a maximum was measured at $185^{\circ} \mathrm{C}$ corresponding well to the beginning of the sharp continuous decrease in remanent strain (Fig. 5). This temperature is lower than the stress-free phase transformation temperature determined by x-ray and permittivity measurements. A decrease in the phase transition temperature due to uniaxial compressive stress perpendicular to the polarization direction, which is the case in mechanically compressed ferroelastic materials, was not expected. It has been shown using a Devonshire thermodynamic formalism that a two-dimensional compressive stress perpendicular to the polarization in a normal FE should increase the Curie temperature. ${ }^{23,24}$ Above this temperature there was a discontinuous decrease in compliance due to the initiation of a transition to the higher temperature $P 4 / \mathrm{mmm}$ potentially ferroelastic phase. With increasing tem-

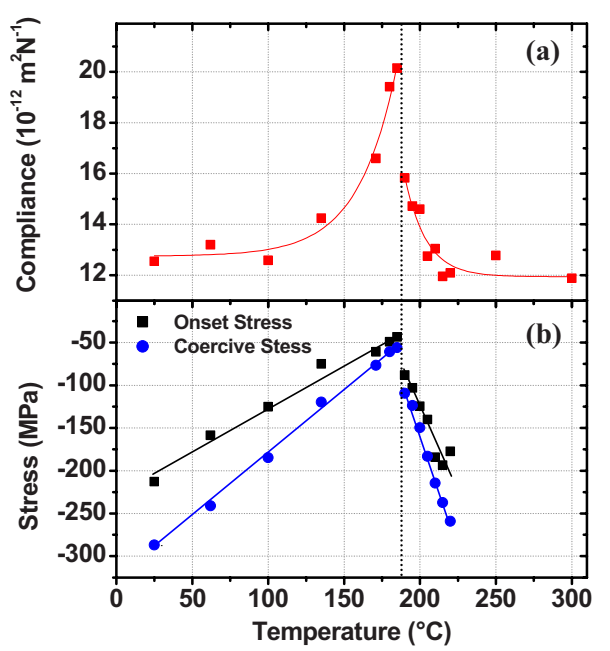

FIG. 6. (Color online) (a) Compliance and (b) characteristic stresses (onset and coercive stress) as a function of temperature for BNT-6BT. The curve fit lines are meant only to guide the eye.

perature there was a subsequent nonlinear decrease in compliance to values comparable to those at room temperature. Previous measurements of the compliance of single crystal BT as a function of temperature around the FE-paraelectric transformation temperature have shown similar results to that presented here. ${ }^{25}$ Because of the measurement technique utilized, it was not possible to separate the intrinsic from the extrinsic reversible contributions, such as reversible domain wall motion. The compliance values presented here are therefore effective values, representing not only the composite intrinsic response of all the domains present but also the effects of nonlinear reversible behavior.

The measured onset and coercive stresses were found to both linearly decrease from room temperature up to $185^{\circ} \mathrm{C}$ at a rate of $1.02 \mathrm{MPa}{ }^{\circ} \mathrm{C}^{-1}$ and $1.48 \mathrm{MPa}{ }^{\circ} \mathrm{C}^{-1}$, respectively. Additionally, the difference between these two characteristic stresses was also found to decrease with temperature, in part due to the increase in the initial effective compliance and the tangent modulus at the coercive stress. Between 185 and $190^{\circ} \mathrm{C}$ there was a discontinuous increase in both stress parameters that coincided with the discontinuity measured in the effective compliance (shown as a dotted line in Fig. 6), which was followed by a linear increase in the characteristic stresses to $220{ }^{\circ} \mathrm{C}$ at a rate of $-3.64 \mathrm{MPa}{ }^{\circ} \mathrm{C}^{-1}$ and $-5.30 \mathrm{MPa}{ }^{\circ} \mathrm{C}^{-1}$, respectively. The difference between the characteristic stress parameters increased with temperatures above $185^{\circ} \mathrm{C}$. Above $220^{\circ} \mathrm{C}$ the maximum applied stress, limited by the capabilities of the load frame, was no longer large enough to drive a nonlinear material response significant enough to determine the coercive stress. The increase in characteristic stresses above $200{ }^{\circ} \mathrm{C}$ corresponds to a decrease in the hysteresis within the closed loop. It is interesting to note that the XRD analysis (Fig. 3) of BNT-6BT at $200{ }^{\circ} \mathrm{C}$ under compressive loading, which showed the appearance of octahedral tilting and asymmetry at the lower angle side of the (200) $)_{p c}$ peak beginning at $-150 \mathrm{MPa}$, corresponds well with the coercive stress measured from macroscopic stress-strain curves. In addition, from Fig. 3(b) it is apparent that $-210 \mathrm{MPa}$, where the satu- 
ration of these effects in the XRD analysis was shown, is approximately where macroscopic measurements show a saturation of the strain response.

There exists a possibility that the observed high temperature closed loop hysteresis in BNT-6BT is due to antiferroelasticity, a concept first introduced in 1969 by Aizu to describe the theoretical stress-induced phase transition from point symmetry $\mathrm{mmm}$ to point symmetry $2 / \mathrm{m}$ at temperature $T_{0}{ }^{26}$ Potentially antiferroelastic single crystals of lithium cesium sulfate $\left(\mathrm{LiCsSO}_{4}\right)$ have been shown around the ferroelastic phase transition temperature $(202 \mathrm{~K})$ to display a double loop behavior as well as a typical ferroelastic behavior, depending on the crystal lattice orientation. ${ }^{27,28}$ This behavior, however, was only observed in response to shear stress. Further experimental results of $\mathrm{LiCsSO}_{4}$ under uniaxial compression did not show an antiferroelastic-like behavior. ${ }^{29}$ Antiferroelasticity is only possible if the cells are equally distorted in an opposite sense, alternately with a zero resultant, ${ }^{30}$ which is a possibility in this material due to the $c / a$ ratio in the higher temperature $P 4 / \mathrm{mmm}$ that is less than one. Just as AFEs can be changed to FEs by an electric field, it is expected that antiferroelastics can be changed to ferroelastics by an applied mechanical stress. The analog in the field of ferroelectricity, antiferroelectricity has been thoroughly studied in $\mathrm{Pb}$-based perovskites, most notably in modifications of PZT close to the lead zirconate (PZ) end member. ${ }^{31}$ A characterizing parameter in antiferroelectricity is the critical electric field for induced ferroelectricity, which depends on composition, ${ }^{32}$ temperature, ${ }^{33}$ and stress state. ${ }^{9}$ However, the electric field required for an AFE-FE phase transition in PZ increases with decreasing temperature leading to the classical double loop polarization-electric field loop only below the AFE-FE phase transition temperature, the opposite of that observed in BNT-6BT.

\section{CONCLUSIONS}

Stress-free XRD analysis on polycrystalline BNT-6BT showed a phase transformation between polar tetragonal $P 4 m m$ and nonpolar tetragonal $P 4 / \mathrm{mmm}$ at $195{ }^{\circ} \mathrm{C}$, well below the Curie temperature. However, the application of a uniaxial compressive stress at $200{ }^{\circ} \mathrm{C}$ initiated a $(211)_{\text {tet }}$ superlattice reflection, characteristic of oxygen octahedral tilting, representing a stress-induced $P 4 / \mathrm{mmm}-\mathrm{P} 4 / \mathrm{bm}$ phase transition at $200{ }^{\circ} \mathrm{C}$. Additional macroscopic uniaxial compressive loading experiments on unpoled polycrystalline BNT-6BT specimens revealed closed loop hysteresis behavior in the vicinity of the stress-free $P 4 m m-P 4 / m m m$ transition temperature. Macroscopic compressive measurements at higher temperatures, well away from both the lower tempera- ture polar phase and the higher temperature cubic phase, have shown a lack of ferroelastic switching in the $P 4 / \mathrm{mmm}$ phase, despite the measured tetragonality.

\section{ACKNOWLEDGMENTS}

This work was supported by the Leibniz program of the Deutsche Forschungsgemeinschaft.

${ }^{1}$ J. Rödel, W. Jo, K. T. P. Seifert, E. M. Anton, T. Granzow, and D. Damjanovic, J. Am. Ceram. Soc. 92, 1153 (2009).

${ }^{2}$ G. A. Smolenskii, V. A. Isupov, A. I. Agranovskaya, and N. N. Krainik, Sov. Phys. Solid State 2, 2651 (1961).

${ }^{3}$ C. Xu, D. Lin, and K. W. Kwok, Solid State Sci. 10, 934 (2008).

${ }^{4}$ T. Takenaka, K. Maruyama, and K. Sakata, Jpn. J. Appl. Phys., Part 133 , 2236 (1991).

${ }^{5}$ J. Suchanicz, K. Roleder, J. Kwapulinski, and I. JankowskaSumara, Phase Transitions 57, 173 (1996).

${ }^{6}$ S. T. Zhang, A. B. Kounga, E. Aulbach, and Y. Deng, J. Am. Ceram. Soc. 91, 3950 (2008).

${ }^{7}$ G. O. Jones and P. A. Thomas, Acta Crystallogr. B 58, 168 (2002).

${ }^{8}$ H. X. Wang, H. Q. Xu, H. S. Luo, Z. W. Yin, A. A. Bokov, and Z. -G. Ye, Appl. Phys. Lett. 87, 012904 (2005).

${ }^{9}$ X. Tan, J. Frederick, C. Ma, E. Aulbach, M. Marsilius, W. Hong, T. Granzow, W. Jo, and J. Rödel, Phys. Rev. B 81, 014103 (2010).

${ }^{10}$ M. I. Morozov and D. Damjanovic, J. Appl. Phys. 104, 034107 (2008).

${ }^{11}$ X. Tan, E. Aulbach, W. Jo, T. Granzow, J. Kling, M. Marsilius, H. -J. Kleebe, and J. Rödel, J. Appl. Phys. 106, 094102 (2009).

${ }^{12}$ J. E. Daniels and M. Drakopoulos, J. Synchrotron Radiat. 16, 463 (2009).

${ }^{13}$ J. E. Daniels, J. Appl. Crystallogr. 41, 1109 (2008).

${ }^{14}$ T. Roisnel and J. Rodríguez-Carvajal, Mater. Sci. Forum 378-381, 118 (2001).

${ }^{15}$ K. G. Webber, E. Aulbach, T. Key, M. Marsilius, T. Granzow, and J. Rödel, Acta Mater. 57, 4614 (2009).

${ }^{16}$ Y. Hiruma, K. Yoshii, H. Nagata, and T. Takenaka, Ferroelectrics 346, 114 (2007).

${ }^{17}$ W. Jo, J. E. Daniels, J. L. Jones, X. Tan, P. A. Thomas, D. Damjanovic, and J. Rödel, In preparation (2010).

${ }^{18}$ V. Dorcet, G. Trolliard, and P. Boullay, Chem. Mater. 20, 5061 (2008).

${ }^{19}$ G. Trolliard and V. Dorcet, Chem. Mater. 20, 5074 (2008).

${ }^{20}$ J. A. Gonzalo and J. M. Rivera, Ferroelectrics 2, 31 (1971).

${ }^{21}$ W. J. Merz, Phys. Rev. 91, 513 (1953).

${ }^{22}$ T. Leist, T. Granzow, W. Jo, and J. Rödel, "Effect of tetragonal distortion on ferroelectric domain switching: A case study on La-doped $\mathrm{BiFeO}_{3}-\mathrm{PbTiO}_{3}$ ceramics," J. Appl. Phys. (in press).

${ }^{23}$ G. A. Rossetti, Jr., L. E. Cross, and K. Kushida, Appl. Phys. Lett. 59, 2524 (1991).

${ }^{24}$ F. Jona and G. Shirane, Ferroelectric Crystals (Pergamon, New York, 1962).

${ }^{25}$ E. J. Huibregtse, W. H. Bessey, and M. E. Drougard, J. Appl. Phys. 30, 899 (1959).

${ }^{26}$ K. Aizu, J. Phys. Soc. Jpn. 27, 1171 (1969).

${ }^{27}$ G. Pakulski, B. Mróz, and T. Krajewski, Ferroelectrics 48, 259 (1983).

${ }^{28}$ J. A. Tuszyńaski, B. Mróz, H. Kiefte, and M. J. Clouter, Ferroelectrics 77, 111 (1988)

${ }^{29}$ G. Pakulski, T. Breczewski, B. Mróz, and T. Krajewski, Ferroelectrics 74, 375 (1987).

${ }^{30}$ M. E. Lines and A. M. Glass, Principles and Applications of Ferroelectrics and Related Materials (Clarendon, Oxford, 1977).

${ }^{31}$ B. Jaffe, W. R. Cook, and H. Jaffe, Piezoelectric Ceramics (Academic, New York, 1971).

${ }^{32}$ G. Shirane, Phys. Rev. 86, 219 (1952).

${ }^{33}$ E. Sawaguchi and T. Kittaka, J. Phys. Soc. Jpn. 7, 336 (1952). 